La alcoba, el lecho, lo cotidiano. Cultura material de un espacio doméstico. Córdoba (Argentina), siglos XVIII y XIX

Cecilia Moreyra

páginas / año 10 - n 24 Septiembre-Diciembre / ISSN 1851-992X/ pp.95-117/

2018

http://revistapaginas.unr.edu.ar/index.php/RevPaginas

\title{
La alcoba, el lecho, lo cotidiano Cultura material de un espacio doméstico. Córdoba (Argentina), siglos XVIII y XIX
}

\author{
Bedroom, bed and everyday life \\ Material culture of a domestic space in Córdoba (Argentina) in the \\ $18^{\text {th }}$ and19th centuries
}

\author{
Cecilia Moreyra \\ Centro de Investigaciones y Estudios sobre Cultura y Sociedad, \\ Consejo Nacional de Investigaciones Científicas y Técnicas, \\ Universidad Nacional de Córdoba (Argentina)
}

\section{Resumen}

En este artículo construimos una historia cultural de un fragmento del espacio doméstico de Córdoba entre finales del siglo XVIII y el siglo XIX: la alcoba y el lecho cotidiano, un lugar multifuncional y multiactoral. Es una historia de la vivienda y de la vida privada, esto significa que observamos la estructura edilicia junto con los objetos y los cuerpos que la habitaban, que, en continua interacción, producían prácticas cotidianas de las que buscamos dar cuenta, describiendo el entorno material e interpretando la presencia o ausencia de determinados objetos y la disposición de cada elemento dentro de la estructura habitacional. Para adentrarnos en este universo de lo ordinario analizamos inventarios post mortem, cartas dotales y testamentos conservadas en el Archivo Histórico de la Provincia de Córdoba, documentos triangulados con relatos de viajeros.

\section{Palabras Clave}

Alcoba; Lecho; Espacio doméstico; Vida Cotidiana; Cultura Material.

\begin{abstract}
In this article we build a cultural history of an element of domestic space in Córdoba between the end of the 18th century and the 19th century: the bedroom and the daily bed, a multifunctional and multiactoral place. It is a history of dwelling and private life, it means, we observe the building structure and the objects and bodies that inhabited it, which, in continuous interaction, produced daily practices that we seek to stud by describing the material environment and interpreting the presence or absence of certain objects and the disposition of each element within the housing structure. To study this ordinary universe we analyze post-mortem inventories, dowry and wills preserved in the Historical Archive of the Province of Córdoba, triangulated documents with travelers' stories.
\end{abstract}

\section{Keywords}

Bedroom; Bed; Domestic space; Everyday life; Material culture. 


\section{La alcoba, el lecho, lo cotidiano. Cultura material de un espacio doméstico. Córdoba (Argentina), siglos XVIII y XIX}

\section{Introducción}

Este artículo explora fragmentos de los interiores domésticos de la ciudad de Córdoba: una habitación, la alcoba y un espacio, el lecho. Proponemos, pues, un recorrido por estos cuartos, de los que observaremos las interacciones habidas entre cuerpos y objetos. El espacio doméstico es la unidad material en la que tienen lugar las prácticas cotidianas; allí habitan personas y conjuntos de personas que, emparentadas o no, comparten un lugar, es decir, conviven. Lo doméstico equivale al interior de la vivienda y lo que allí tiene lugar. Se trata de un espacio cuyas formas materiales y simbólicas cambiaron con el tiempo, tendiendo, durante el período que aquí observamos, a especializarse y privatizarse, es decir, a constituirse en lugar separado y opuesto al espacio público.

Nuestra mirada del espacio doméstico, se acota a la ciudad de Córdoba en un espectro temporal amplio, que se extiende desde finales del siglo XVIII hasta avanzada la siguiente centuria. Se trata de un período complejo, signado por procesos de distinto orden, que no solo incluyen el espacio cordobés, sino también, el rioplatense y el latinoamericano. La desestructuración del orden colonial, a comienzos del siglo XIX, implicó enfrentamientos bélicos y la desarticulación de algunos circuitos comerciales. Esto significó, para Córdoba, la reorientación del comercio exterior -antes orientado al Alto Perú- hacia Buenos Aires y el mercado atlántico. Asimismo, la región rioplatense se integró al área mercantil externa como mercado consumidor de los productos industrializados de Gran Bretaña. Aun ocupando un espacio periférico, la ciudad de Córdoba estaba estratégicamente situada en el nodo de las rutas comerciales que conectaban el puerto de Buenos Aires con el norte de las Provincias Unidas, así como con la región de Cuyo y Chile. Como "lugar de paso", Córdoba era transitada por inmigrantes y comerciantes, junto con objetos, ideas y prácticas. Todo ello se mixturaba con modos y formas locales, generando la convergencia de elementos culturales tradicionales y modernos, nativos y foráneos.

Frente a las variaciones y rupturas manifiestas en los ámbitos político, económico, comercial y social, nos preguntamos si el universo de lo cotidiano se hizo eco de algunos de esos cambios, ¿experimentaron variaciones las prácticas ordinarias, así como los espacios y objetos que las constituían? ¿tendieron las formas domésticas a permanecer casi inalterables durante el período observado? Pensamos que indagar un fragmento del espacio doméstico en un dilatado período, habilita la identificación de rupturas y continuidades en las prácticas cotidianas y en las formas de estructurar el espacio doméstico que es, en definitiva, una forma de organizar los cuerpos. En esta línea, suscribimos a la propuesta de Michelle Perrot, quién concibe la alcoba como un microcosmos, un átomo, conveniente para este tipo de lecturas, ya que remite a todo aquello de lo cual forma parte y de lo que es partícula 


\section{Cecilia Moreyra}

elemental $^{1}$. La historiadora francesa realizó una exhaustiva investigación sobre la habitación occidental, específicamente, francesa. Frente a tamaña obra precursora, la presente es una contribución a la historia de un espacio doméstico en los márgenes de ese mundo occidental, en los confines del espacio latinoamericano.

Para adentrarnos en el mundo espacial y material ordinario de la Córdoba de los siglos XVIII y XIX, analizamos un conjunto de inventarios postmortem conservados en la serie "Escribanías" del Archivo Histórico de la Provincia de Córdoba. Estos documentos se elaboraban luego de un fallecimiento y tenían la finalidad de contabilizar los bienes que habrían de repartirse entre los herederos. Los registros son exhaustivos, en ellos constan tamaño, material, color, ornamentos, estado de conservación y valor económico de las viviendas, mobiliario y enseres varios. Dentro de la masa documental disponible, seleccionamos un total de doscientos inventarios realizados entre 1778 y 1870 . Las fuentes seleccionadas fueron aquellas que contienen datos significativos de las viviendas y sus objetos, necesarios para el análisis propuesto. Ante las extensas nóminas de objetos que encontramos en los inventarios, sistematizar se volvió imperioso para organizar la información y cuantificar objetos específicos, por ejemplo, muebles de las alcobas o ropa de cama, determinando su frecuencia a lo largo del tiempo. Esta operación permitió identificar la cantidad de veces que un objeto preciso aparece mencionado en el conjunto de inventarios consultados y sus variaciones a lo largo del tiempo. Los datos relevados de los inventarios fueron triangulados con los provistos por otras fuentes: cartas dotales, testamentos y relatos de viajeros.

Al dirigir la mirada al mundo de lo cotidiano se incurre en el desacierto de "dar por sentado" muchas cosas, presuponer que lo que hoy en día se entiende por alcoba, cama o reposo, decanta por su obviedad, "todo el mundo" lo sabe. Es aquí donde la mirada del historiador debe volverse particularmente inquisitiva, cuestionando la lógica práctica, desnaturalizando los espacios, objetos y prácticas cotidianas y trascendiendo el sentido común, adoptando una postura de extrañamiento en el sentido antropológico del término, es decir, convirtiendo lo familiar en exótico ${ }^{2}$. Para superar el pensamiento convencional, reivindicamos la labor descriptiva como método nodal a la hora de conocer y explicar el pasado cotidiano. Howard Becker sugiere la "descripción masiva" como ejercicio metodológico que da cuenta de un fenómeno, un escenario, una persona o un grupo, en forma minuciosa, detallada y directa, es decir, sin hacer interpretaciones. Habiendo aceptado que una descripción libre de interpretaciones es inexistente, ya que cualquier descripción requiere selección y refleja un punto de vista, podemos avanzar en describir un estado de

\footnotetext{
${ }^{1}$ Michelle Perrot. Historia de las Alcobas. México, Fondo de Cultura Económica, 2011, p. 14.

${ }^{2}$ Gustavo Lins Ribeiro. "Descotidianizar. Extrañamiento y conciencia práctica, un ensayo sobre la perspectiva antropológica", en Mauricio Boivin, Ana Rosato y Victoria Arribas (editores) Constructores de otredad. Una introducción a la antropología social y cultural. Buenos Aires: Eudeba, 1998, pp. 194-198.
} 


\section{La alcoba, el lecho, lo cotidiano. Cultura material de un espacio doméstico. Córdoba (Argentina), siglos XVIII y XIX}

cosas hasta agotar los detalles más ínfimos, lo "infraordinario", evitando elaborar resúmenes analíticos de lo observado o leído ${ }^{3}$.

La presencia de cuartos designados como alcobas o dormitorios era, en la ciudad de Córdoba, propia de las viviendas que tenían varios ambientes, como las casas a patios. Con todo, esas alcobas distaban de funcionar como lugares exclusivos del descanso nocturno, pues, como veremos, allí tenían lugar otras prácticas cotidianas. Ello hacía de estas habitaciones, lugares multifuncionales y multiactorales. Por su parte, las casas más pequeñas y los ranchos, formados por apenas una o dos habitaciones, carecían de espacios particulares para dormir: el lecho cotidiano era provisorio y, en muchos casos, también precario.

En nuestro análisis de las alcobas, convergen varias líneas de trabajo: la historia de la vivienda, la de la vida privada y cotidiana y la de la cultura material; espacios de investigación que cristalizan a partir del giro antropológico que dieron las ciencias sociales, en general y la historia, en particular, entre las décadas del 70 y 80 del siglo pasado. Al adentrarnos en esta habitación nos concentraremos en su materialidad: sus cuatro paredes, techo, suelo, puertas y ventanas, y los objetos que la habitaron: camas, baúles, cómodas y escritorios. El conjunto todo conforma un escenario particular que producirá comportamientos y prácticas específicos.

\section{Una mirada histórica de la cultura material y la vida cotidiana}

Los objetos nos rodean, más aun, nos constituyen. Somos una amalgama de lo natural y lo artificial. Un objeto es una entidad física y tangible que fue imaginada, construida, nombrada, deseada, vendida, comprada, utilizada, compartida y tal vez, termine desechada o reutilizada. Estudiar la cultura material es analizar las dimensiones físicas de los objetos, es decir, tamaño, material, color o estilo, como una vía para aproximarse al pensamiento y acción humanos ${ }^{4}$. Las cosas, aparentemente inanimadas, actúan sobre las personas $\mathrm{y}$, a su vez, son utilizadas por éstas, para el desarrollo de diferentes prácticas cotidianas, regular relaciones y dar significado y sentido a la actividad humana ${ }^{5}$. La cultura material es una temática multidimensional que reclama análisis desde la arqueología, geografía, arquitectura, arte, diseño, antropología e historia. Desde los trabajos fundacionales de Fernand Braudel, ${ }^{6}$ Daniel Roche, ${ }^{7}$ Arjun Appadurai, ${ }^{8}$ y Sindey Mintz, ${ }^{9}$ los historiadores vienen

\footnotetext{
${ }^{3}$ Howard Becker. Trucos del oficio. Cómo conducir su investigación en ciencias sociales. Buenos Aires, Siglo XXI Editores, 2009.

4 Henrry Glassie. Material culture. Bloomington/Indianapolis, Indiana University press, 1999, p. 41.

5 Ian Woodward. Understanding material culture. Londres, SAGE Publications, 2007.

${ }^{6}$ Fernand Braudel. Civilización material, economía y capitalismo, siglos XV-XVIII. 1. Las estructuras de lo cotidiano. Madrid, Alianza, 1984.

${ }^{7}$ Daniel Roche. La Culture des apparences: essai sur l'histoire du vêtement aux SS. XVII y XVIII. París, Fayard, 1989.

${ }^{8}$ Arjun Appadurai (Editor). La vida social de las cosas. Perspectiva cultural de las mercancías. México, Grijalbo, 1991.

${ }^{9}$ Sindey Mintz Dulzura y poder. El lugar del azúcar en la historia moderna. México, Siglo XXI editores, 1996.
} 


\section{Cecilia Moreyra}

observando a objetos y mercancías como actores y motores de los procesos históricos.

Cultura material es el concepto convencionalmente utilizado en el estudio de los objetos y artefactos en interacción con las personas. Arnold Bauer lo define como el conjunto de cosas que hombres, mujeres y niños producen y usan para comer, cubrirse, morar y trabajar ${ }^{10}$. En su investigación, pionera dentro de la historia de la cultura material en Latinoamérica, se demuestra que investigar los precios o la oferta y demanda no logra explicar el consumo de determinado alimento, ropa o abrigo, sino que es necesario bucear en el ámbito de la cultura para entender que también se consume como expresión de individualidad o identidad étnica, cultural y de clase.

El referido giro cultural que dieron las ciencias sociales implicó un viraje hacia lo cultural, lo simbólico y un retorno al sujeto. La investigación histórica se acercó a la antropología, de la que tomó prestados conceptos y métodos, ampliando, de este modo, su objeto de estudio hasta provocar una multiplicación de temas de investigación. Todo, o casi todo, podía ser objeto de estudio de la historia, y lo íntimo y privado también fue puesto bajo la lupa del historiador. En este marco nacieron textos fundamentales como la obra colectiva Historia de la vida privada que dirigieran Philippe Aries y Georges Duby ${ }^{11}$. Allí se vislumbra una Edad Media donde paulatinamente se abandona la gregariedad del ambiente común para descubrir la interiorización del espacio doméstico que resguardaba lo más preciado y secreto, ya fueran bienes, alimentos, hijas, hijos o sirvientes. El proceso de privatización e individualización del espacio cotidiano será largo, y recién entre las sociedades burguesas el concepto de privacidad adquirirá verdadero sentido. La idea de espacio doméstico privado, íntimo y secreto cristalizará en una habitación particular: la alcoba. Es allí donde dirigirá su mirada Pascal Dibie en su Etnología de la Alcoba ${ }^{12}$, obra que manifiesta ese cercano vínculo entre historia y antropología. Su objetivo fue construir una historia del espacio que las personas destinaron al reposo, desde los más pretéritos tiempos hasta la actualidad. Claro que no solo se trata del reposo nocturno, sino de las múltiples prácticas que se fueron gestando en torno a la cama. El recorrido es espacial y temporalmente ambicioso y ofrece un panorama acabado de este espacio doméstico que hoy vinculamos a lo íntimo y privado.

La habitual asociación del dormitorio con las ideas de intimidad y privacidad requiere repensarse y, para ello, historizarse. Un camino posible es volver nuestra mirada a la Europa medieval, en la que dormir y levantarse eran funciones que no se habían privatizado por completo ni se las había excluido de la vida social. Más aún, se acostumbraba recibir visitas en los espacios donde se dormía durante las noches. En aquella época, muy lejos se estaba aún de la privatización e

\footnotetext{
10 Arnold Bauer. Goods, Power, History. Latinamerica's material culture. Nueva York, Cambridge University Press, 2001.

${ }_{11}$ Philippe Aries y Georges Duby (Directores). Historia de la vida privada, 5 tomos. Barcelona, Turus Minor, 2001.

12 Pascal Dibie. Etnología de la Alcoba. El dormitorio y la gran aventura del reposo de los hombres. Barcelona, Gedisa, 1989.
} 


\section{La alcoba, el lecho, lo cotidiano. Cultura material de un espacio doméstico. Córdoba (Argentina), siglos XVIII y XIX}

individualización de la alcoba, era frecuente que varias personas pasaran la noche en la misma habitación, el señor con sus criados y la señora con sus doncellas; en los otros grupos sociales también se compartía el espacio y la experiencia. Así, solían dormir juntos, no solo en la misma habitación sino en la misma cama, hombres y mujeres, viejos y niños, dueños de casa y huéspedes. ${ }^{13}$ Ese carácter público y privado de la alcoba se expresa notoriamente en la cámara de Luis XIV, en la que el acostarse y levantarse del rey eran verdaderos actos políticos. Cargada de profundo significado simbólico, la habitación real se encontraba en el centro mismo de un sistema de poder. Reglas, límites y rituales rodeaban este espacio en el que nada se dejaba librado al azar, sino que cada acto formaba parte de una cuidada organización. No en el sentido moderno del término, sino que cada acto estaba investido de prestigio, acorde a los actores intervinientes dentro del esquema de distribución de poder. ${ }^{14}$

Interesada en la historia social de la vivienda, la historia de la vida privada y la historia de las mujeres, Michelle Perrot ${ }^{15}$ encontró en las alcobas el punto de convergencia de esas líneas de investigación. Se valió de fuentes literarias, tales como tratados de arquitectura o de artes decorativas, revistas especializadas en mobiliario, manuales de higiene, investigaciones médicas y sociales sobre el hábitat, diarios de viajes, literatura personal, novelas y poesías; asimismo, algunas pinturas, grabados y fotografías complementan su análisis. Perrot se concentra en el proceso de transformación del dormitorio común en la habitación privada, máxima expresión de individualidad del sujeto moderno. En este punto también insiste Rafaella Sarti al poner de manifiesto la concurrencia y promiscuidad de las camas hasta bien avanzado el siglo XVIII'16. La historiadora italiana reconoce en la cama un objeto de ostentación entre las clases altas, un espacio de abrigo contra los intensos inviernos europeos y un artefacto que con el tiempo fue multiplicándose en número y especializándose en sus funciones.

El dormitorio experimentó, en gran parte de Europa, notables transformaciones durante el siglo XVIII, período que Joan Dejean denomina "la era del confort", 17 cuyas características distintivas serán la multiplicación, especialización y reubicación del mobiliario. Cada vez más muebles poblando las casas, muebles que se destinaban a funciones específicas y artefactos que dejaban su carácter móvil o "mueble", para asentarse en una habitación particular de la casa. Pequeños grandes cambios que significaron nuevas formas de sociabilidad y cambios en las prácticas cotidianas que tendieron a producir experiencias corporales más confortables.

\footnotetext{
${ }^{13}$ Norbert Elias. El proceso de la civilización. Investigaciones sociogenéticas y psicogenéticas. México, Fondo de Cultura Económica, 2009, $3^{\circ}$ edición, p. 252.

${ }_{14}$ Norbert Elias analiza las ceremonias que tenían lugar en el dormitorio de Luis XIV haciendo inteligibles a través de ellas la estructura y el funcionamiento de la configuración cortesana de la que eran parte. La sociedad cortesana. México, Fondo de Cultura Económica, 2012, pp. 108-152.

15 Michelle Perrot. Historia de las alcobas...Op. Cit.

${ }^{16}$ Rafaella Sarti. Vida en familia. Casa, comida y vestido en la Europa moderna. Barcelona, Crítica, 2003.

${ }^{17}$ Joan Dejean. The Age of comfort. When Paris Discovered Casual and the Modern Home Began. Nueva York, Bloomsbury USA, 2010.
} 


\section{Cecilia Moreyra}

En América Latina, algunos investigadores coinciden en situar el proceso de especialización de las estancias de la casa entre finales del siglo XVIII y mediados del XIX. Este desarrollo habría implicado la privatización de ciertas habitaciones, como los dormitorios, que estarían separados de espacios más públicos. En Santiago de Cuba, por ejemplo, Aida Morales Tejeda sitúa la cristalización de este proceso de especialización de las piezas de la vivienda, en el cambio del siglo XVIII al XIX. En este marco, la sala, por ejemplo, se erigió como el espacio para recibir visitas; el estudio, para los caballeros; el tocador para las damas y el sitio para dormir deja de ser una simple "habitación" para constituirse en cámara. Allí, fue la cama el mueble destacado que acompaño la introducción de variado mobiliario de influencia francesa ${ }^{18}$. En un período equivalente, Arnaldo Moya Gutiérrez interpreta la tendencia hacia la especialización de los distintos aposentos de las casas de San José de Costa Rica, como una búsqueda de intimidad ${ }^{19}$. Por su parte, en la ciudad de México, María Esther Pérez Salas extiende el fenómeno desde finales del siglo XVIII hasta mediados del XIX. Es en este período cuando se organizaron los interiores domésticos mediante la división de espacios "públicos", como sala u oratorio y los "privados" o íntimos como los dormitorios ${ }^{20}$. En el Zacatecas colonial, Francisco García Gonzalez introduce un matiz esencial vinculado a las diferencias sociales y económicas de sus habitantes. Así, además de las esperadas diferencias en términos de tamaño y materiales, lo significativo radicaba en la independencia, y con ello, intimidad, de las habitaciones, característica propia de las casas de familias acomodadas. Diferente era la situación en las denominadas casas medias, donde se combinaban los espacios para la habitación familiar y el trabajo. Ya en las casas bajas, la mixtura de actividades era aún más profunda: en una misma habitación se comía y se dormía 21.

\section{Los espacios}

La arquitectura doméstica tiene capacidad discursiva ${ }^{22}$, es un texto en el que podemos leer prácticas, interacciones, sensibilidades y relaciones de poder. El espacio construido condiciona, a la vez que es condicionado por los vínculos sociales. Así, el tamaño de la casa, los materiales con que está construida, el número

\footnotetext{
${ }^{18}$ Aida Morales Tejeda. El signo francés en Santiago de Cuba. Santiago de Cuba, Editorial Oriente, 201, pp. 94, 127-138

19 Arnaldo Moya Gutiérrez. "Cultura material y vida cotidiana: el entorno doméstico de los vecinos principales de Cartago (1750-1820)” en Molina Jiménez, Iván y Palmer, Steven (Ed) Héroes al gusto y libros de moda. Sociedad y cambio cultural en Costa Rica (1750/1900), San Jose, Costa Rica, Ed. Porvenir, Plumsock Mesoamerican Studies, 1992, p. 22

${ }^{20}$ María Esther Pérez Salas, "El trajín de una casa" en Gonzalbo Aizpuru Pilar (Dir): Historia de la vida cotidiana en México Vol. IV: Bienes y vivencias. El siglo XIX, El Colegio de México y FCCE, México DF, 2005 , p. 182.

21 Francisco García González, "Vida cotidiana y cultura material en el Zacatecas colonial" en Gonzalobo Aizpuru, Pilar (dir): Historia de la vida cotidiana en México vol III el siglo XVIII: entre tradición y cambio, FCE, México, 2005, p. 53.

22 Enrique Ayala Alonso. "Habitar la casa barroca. Una experiencia en la ciudad de México" Actas del III Congreso Internacional del Barroco, 2001.
} 


\section{La alcoba, el lecho, lo cotidiano. Cultura material de un espacio doméstico. Córdoba (Argentina), siglos XVIII y XIX}

de habitaciones, su estética y el lugar que ocupa dentro de la ciudad influyen en las prácticas y vínculos que en él se desarrollan. El espacio habitable, dice Gloria Franco, es un hecho social y cultural por lo que es menester analizar no solo características físicas sino, principalmente, el valor simbólico de la casa ${ }^{23}$. En esta misma línea, en su investigación sobre las viviendas del Buenos Aires virreinal, Osvaldo Otero concibe al espacio cotidiano, los objetos, formas y elementos decorativos, como un todo que permite comprender las conductas y las formas recíprocas de interacción entre los hombres. ${ }^{24}$

En la ciudad de Córdoba se alzaban espaciosas viviendas a patios así como pequeñas casitas o ranchos de una o dos habitaciones. Las primeras estaban organizadas en torno tres patios: el principal, el secundario y el corral. El primero era el eje estructurador de los espacios más importantes: la sala de recibo, el zaguán, las alcobas de la familia, el comedor y las tiendas y trastiendas. Estas habitaciones se ubicaban alrededor del patio y estaban comunicadas con éste y sus galerías, a través de puertas y ventanas. El segundo patio se alzaba como punto central de los espacios de servicio, integrados por cocina, despensa, horno de pan, pozo de agua y lugares comunes. Finalmente, en el traspatio, estaba el corral para los animales y la huerta; estos lugares conformaban el sector periférico de la vivienda, "el fondo" de la casa. Edificios como éstos eran habitados, especialmente, por familias acomodadas, conformadas por personas de etnia blanca, es decir, españoles peninsulares y americanos, cuyos nombres iban precedidos por el apelativo "Don-Doña". El grueso de esta elite urbana se dedicaba al comercio de importación y exportación; otro tanto, eran profesionales, hacendados, religiosos y militares de alto rango. Propietarios de tierras, esclavos, animales, inmuebles, apellido y capital social, tenían, a su vez, alta participación en el poder político local.

En las viviendas como la que describimos, la habitación destinada al descanso nocturno se registró con el nombre de alcoba o "cuarto que sirve de dormitorio" y se situaba en torno al patio principal, ocupando un lugar entre los cuartos más importantes de la casa. Esta disposición espacial vislumbra la inexistencia de una separación taxativa entre cuartos públicos, como la sala de recibo, y las alcobas; por el contrario, éstas eran, en general, adyacentes de las primeras y estaban vinculadas a partir de una puerta común. Así se observa en la vivienda del sombrerero Juan Borton, en la que una puerta comunicaba el dormitorio con sala. ${ }^{25}$ Del mismo modo, en la residencia del escribano Bartolomé Matos de Acevedo, la única habitación identificada como dormitorio se hallaba inmediatamente después de la sala principal. ${ }^{26}$ En su tránsito por Córdoba, el naturalista alemán Hermann Burmeister

\footnotetext{
${ }^{23}$ Gloria Franco Rubio. "La vivienda en el Antiguo Régimen. De espacio habitable a espacio social" Chronica Nova $\mathrm{N}^{\circ} 35$, Granada, 2009, pp.63-103.

24 Osvlado Otero. La vivienda porteña en tiempos virreinales, La vivienda porteña en tiempos virreinales. Materiales, uso, función y valor simbólico, Tesis de doctorado en Historia. La Plata, Universidad Nacional de La Plata, 2004, pp. 35, 36.

25 "Inventario de Juan Borton", Córdoba, 1820, Archivo Histórico de la Provincia de Córdoba, Escribanía (esc.) 1, legajo (leg.) 453, expediente (exp.) 13, f. 1v.

26 "Sucesorio de Bartolomé Matos de Acevedo", Córdoba, 1824, AHPC, esc. 2, leg. 119, exp.2, f. 32.
} 


\section{Cecilia Moreyra}

reparó en la disposición interna de algunas residencias de la ciudad que se conservaban "del tiempo de los españoles". En estas viviendas, la alcoba lindaba con la sala principal: "al lado de la sala estaban las habitaciones, la del dueño de casa por lo general". ${ }^{27}$

La sala, dormitorios y demás habitaciones estaban comunicadas entre sí; las puertas de la alcoba la conectaban con el patio y con las habitaciones lindantes, es decir que, para pasar de una habitación a otra, había que salir al patio y de allí ingresar a otro cuarto, o bien, atravesar las habitaciones que se encontraran en medio. Esto implicaba que cualquier actividad llevada a cabo en los diferentes cuartos, incluido el dormitorio, podía quedar interrumpida por el paso de alguien. Las puertas y ventanas del dormitorio que daban al patio, dejaban entrar algo de luz a la habitación, mientras que las alcobas que se encontraban en los sectores más internos de la casa, alejadas del patio, tenían la negativa singularidad de ser poco luminosas. Esto último ocasionaba que fueran avaluadas en menor precio que aquellas más iluminadas. Tal es el caso de uno de los dormitorios de la vivienda del comerciante Antonio Benito Fragueiro, que fue tasado en menor precio que otras habitaciones por ser "escaso de luz" y estar "mui en el interior".28

La iluminación de la alcoba era, efectivamente, apreciada, mientras que la ventilación, por su parte, era valorada, sobre todo, en la teoría: airear el cuarto diariamente era clave para mantener la higiene y salud del cuerpo. Así lo estipulaba el discurso higienista del siglo XIX, del que se hizo eco Manuel Carreño en su Manual de urbanidad y buenas maneras, donde se impelía a mantener la ventilación de los aposentos, para liberarlo de las "exhalaciones de los cuerpos durante la noche", que impregnaban el ambiente; acciones imprescindibles para la "conservación de la salud". ${ }^{29}$ Más que confirmar la periódica ventilación de los cuartos, la inclusión de este precepto en un manual de urbanidad, evidencia lo inusual de esta práctica. Evitar la entrada de aire en un cuarto era síntoma de la persistencia de las tradicionales concepciones entorno a la salud/enfermedad, según las cuales, envolverse y taparse, así como cerrar la habitación, impedían el contacto de la piel con el aire corrupto ${ }^{30}$.

\footnotetext{
${ }^{27}$ Hermann Burmeister. Viaje por los Estados del Plata. Buenos Aires, 1944 en Carlos Segreti, Córdoba. Ciudad y provincia siglo XVI-XX. Según relatos de viajeros y otros testimonios. Córdoba, Junta Provincial de Historia de Córdoba, 1973, p. 414.

28 "Inventario de Antonio Benito Fragueiro", Córdoba, 1813, AHPC, Esc. 1, Leg. 442, Exp. 1, f. 8.

${ }^{29}$ Manuel Carreño. Manual de urbanidad y buenas maneras, para uso de la juventud de ambos sexos, en el cual se encuentran las principales reglas de civilidad y etiqueta que deben observarse en las diversas situaciones sociales, precedido de un breve tratado sobre los deberes morales del hombre. Córdoba, Buena Vista, 2010, p. 28. Este tipo de literatura circuló en todo el espacio latinoamericano; en la ciudad de Córdoba, por ejemplo, identificamos la presencia de un libro de similar temática, denominado "Código de Urbanidad" en manos de Félix María Olmedo, Córdoba, 1879, AHPC, Esc. 1, Leg. 568, Exp. 14, F.12.

30 Cecilia Moreyra. "Cultura material e higiene cotidiana en la Córdoba del Ochocientos". Anuario de Estudios Americanos Vol. 74, N 1, Sevilla, 2017, pp. 211-234; Cfr. Alain Corbin. El perfume o el miasma. El olfato y lo imaginario social. Siglos XVIII y XIX. México, Fondo de Cultura Económica, 2002.
} 


\section{La alcoba, el lecho, lo cotidiano. Cultura material de un espacio doméstico. Córdoba (Argentina), siglos XVIII y XIX}

En cuanto a su forma, las alcobas eran, en general, cuadradas o ligeramente rectangulares y medían, en promedio, entre cinco y seis varas ${ }^{31}$ de largo por lo mismo de ancho. Considerables dimensiones que permitían la instalación de varios muebles. En la alcoba del comerciante Bernabé Las Heras, por ejemplo, que superaba los veinticuatro metros cuadrados, tenía lugar una cuja matrimonial con pilares, colgadura y cortinas; un catre y dos bancos para armar catres; tres papeleras de diferentes tamaños; un escritorio con tapa; dos mesitas pequeñas; dos baúles; una caja para guardar la ropa blanca; ocho sillas; dos frasqueras y varios cuadros. ${ }^{32}$ Más aún, dentro del dormitorio principal se ubicaba también el despacho del dueño de casa, sitio que servía a los fines de organizar y ejecutar su actividad comercial. Se trata, entonces, de una alcoba de notables proporciones y poblada de numerosos objetos; su carácter multifuncional se evidencia en la diversidad de los muebles que la habitaban: la cuja matrimonial para dormir convivía con escritorios, sillas y papeleras que conformaban el despacho del comerciante. Así pues, esta habitación funcionaba como lugar del reposo nocturno, pero también constituía un sitio de trabajo. Ahora bien, la coexistencia de funciones que vemos en la alcoba del comerciante Las Heras, no necesariamente formaba parte de las experiencias cotidianas de todas familias de similar extracción socio-profesional. En la residencia del comerciante Hilarión Funes, por ejemplo, el despacho comercial ocupaba un lugar específico, una habitación particular, estratégicamente ubicada junto a los almacenes, barracas y prensas de enfardar ${ }^{33}$. En este "cuarto escritorio" se llevaba el papeleo y documentación vinculadas a las actividades productivas y mercantiles de la familia. La existencia de esta pequeña habitación manifiesta un mayor grado de especialización de los espacios y las practicas, es decir, la separación espacial de unas actividades de otras.

En las viviendas a patios como las que venimos observando, los espacios estaban jerarquizados. Las habitaciones se disponían en orden decreciente de importancia: espacios principales, primero y de servicio, detrás. La clasificación del ambiente doméstico se basaba, según Osvaldo Otero, más que en la función, en el usuario ${ }^{34}$, esto quiere decir que, los espacios de servicio eran habitados y frecuentados, principalmente, por esclavos, criados o sirvientes, quienes dormían en los cuartos que estaban adyacentes a la cocina, ${ }^{35}$ cuando no era la misma cocina su lugar de descanso nocturno. Las diferentes categorías de las estancias de la casa, no solo se

\footnotetext{
31 La vara española es una medida de distancia que equivale a 0,8359 metros. Luis García Montes. "Medidas antiguas: la vara." Toletum: boletín de la Real Academia de Bellas Artes y Ciencias Históricas de Toledo $\mathrm{N}^{\circ} 27$, Toledo, 1991, p. 154.

32 “Testamentaria de Bernabé Las Heras", Córdoba, 1821, AHPC, Esc. 3, Leg. 69, Exp.1, f. 78. Fue posible identificar cada uno de los muebles que componían la alcoba ya que el tasador especificó cuidadosamente los objetos que se encontraban en la sala y los que estaban en el dormitorio.

33 Testamentaria de Mercedes Roldán de Funes (viuda de Hilarión Funes), AHPC, Escribanía 1, Año 1873, Leg. 186, Exp. 1.

${ }^{34}$ Osvaldo Otero, La vivienda porteña... Op. Cit.

35 En el segundo patio de la vivienda del comerciante Felipe Antonio González se ubicaban en forma contigua, la despensa, la cocina y "dos cuartos para criados." "Testamentaria de Felipe Antonio González”, Córdoba, 1818, AHPC, Esc. 4, Leg. 51, Exp. 13, f. 2v.
} 


\section{Cecilia Moreyra}

advierte por su disposición dentro del conjunto habitacional o los usuarios de cada uno, sino también, por los materiales con que estaban construidos y el confort de los mismos. Los cuartos de los espacios de servicio solían tener sus pisos de tierra, por ejemplo, a diferencia de las habitaciones principales cuyos pisos eran enladrillados o de baldosa.

En cuanto a las casas de menores dimensiones y ranchos, éstos eran los lugares donde vivían, en general, los individuos y grupos familiares de los sectores subalternos. Esto es, personas que en registros parroquiales y censales eran clasificadas como "pardas" "mulatas" o "negras"; categorizaciones étnicas que, durante el siglo XIX, perdieron vigencia, aunque sí se mantuvieron clasificaciones distintivas en el orden de lo social, como "noble" o "plebeyo"36. Estas personas de los sectores menos favorecidos, se dedicaban a oficios artesanales, al trabajo en pequeños comercios, algunas eran costureras o bien, se desempeñaban en el servicio doméstico. En sus viviendas, las funciones de cada habitación eran poco específicas. Se mencionan, en forma genérica, algunos "cuartos" o "piezas" que no superaban el par. En tales casas, era escasa la separación de actividades según los espacios, y un mismo cuarto podía ser escenario del reposo nocturno, el trabajo diario y la preparación de los alimentos. La costurera viuda María del Tránsito Camargo, por ejemplo, habitaba una casa compuesta por una sala de unos treinta metros cuadrados aproximadamente, en la que había dos mesas, cuatro sillas, una cama, una caja y sus herramientas de trabajo: un devanador y cuatro peines de tejer lienzo. ${ }^{37}$ El lugar para cocinar se conformaba a partir de una "ramadita" que tenía lugar en el patio. Ciertamente, múltiples actividades tenían lugar en un mismo espacio: en la sala se comía, se trabajaba y, a su vez, se dormía. La multifuncionalidad de las habitaciones y la limitada sectorización de las mismas, se vuelve aún más notoria en las viviendas de menores dimensiones y con pocas habitaciones. En las residencias a patios, con numerosos cuartos, se observa una organización espacial que categorizaba los espacios según su importancia social: división de los espacios principales de los de servicio. Pero, aun así, dentro de ciertas habitaciones, como la misma alcoba, podían coexistir múltiples actividades.

\section{El mobiliario}

La cama era el mueble por excelencia de las alcobas, allí se recostaban los cuerpos durante la noche o la siesta; ${ }^{38}$ allí se nacía, allí se enfermaba y allí se moría. La cama era también, espacio de la intimidad sexual practicada así dentro como fuera de la

\footnotetext{
${ }^{36}$ Estas categorías se observan, específicamente, en el empadronamiento de la provincia de Córdoba de 1840.

37 “Inventario de María del Tránsito Camargo", Córdoba, 1813, AHPC, Esc. 1, Leg. 442, Exp. 11, f. 1.

${ }^{38}$ Con respecto a la siesta decía José Manuel Eizaguirre que avanzado el siglo XIX los cordobeses seguían durmiendo las prolongadas siestas coloniales de hasta cinco horas, costumbre considerada por él mismo como "símbolo de atraso y manifestación de muerte" José Manuel Eizaguirre. Córdoba. Primera serie de cartas sobre la vida y costumbres en el interior. Córdoba, R. Bruno y Ca. Editores, 1898, pp. 135, 136.
} 


\section{La alcoba, el lecho, lo cotidiano. Cultura material de un espacio doméstico. Córdoba (Argentina), siglos XVIII y XIX}

institución matrimonial. Las cujas matrimoniales tenían pilares que sostenían un techo o cielorraso de cuero o tela y cortinas que, al correrse, conformaban un nuevo habitáculo, separado y específico, dentro de una misma habitación. Ello permitía construir una intimidad precaria, ocultando los que ocurría detrás de las cortinas. Los biombos también servían a los fines de conformar nuevos espacios, que escondían lo que estaba detrás.

El carácter de intimidad y privacidad que intentaba atribuirse a la alcoba se evidencia en tratados de urbanidad como el ya citado de Manuel Carreño. Allí se establecía un orden deseable de los cuartos, donde sala de recibo y alcoba jamás debían estar una junto a la otra $\mathrm{y}$, dado el caso que fueran colindantes, era importante cuidar que las camas no estuvieran jamás a la vista ya que "el tálamo nupcial, ofrecido a las miradas de los que entran a la sala, no podrá menos que considerarse para las personas cultas y juiciosas, como un signo de vulgaridad y mala educación". ${ }^{9}$ Reglas como éstas se hacían eco de la idea burguesa de privacidad, donde la casa, y en particular el dormitorio, debían estar ocultos. ${ }^{40}$ "El hogar es la quintaesencia del mundo burgués" escribía Eric Hobsbawm, ${ }^{41}$ y tras los muros de la casa tenía lugar el dominio de lo privado, valor primordial y pilar del orden social burgués.

Además de lugar de descanso y espacio de ejercicio de la sexualidad, la cama se constituía en escenario de otras prácticas que tuvieron a las mujeres como protagonistas: en las camas se paría y en las camas muchas mujeres recibieron maltratos. Así le ocurría a Gabriela Cabrera, cuyo marido guardaba debajo de la almohada un cuchillo para amedrentarla cada noche y lograr, de ese modo, que ésta cumpliera con sus deberes de esposa. ${ }^{42}$ Era la cama, también, el lugar donde se atravesaba una enfermedad y se moría, era el "lecho de muerte." Sabemos que muchas personas dictaban su testamento estando "enfermas en la cama", dolencia que, en muchas ocasiones, terminaba en defunción. Era, pues, el lecho, un escenario revestido de cierta solemnidad ya que en él se manifestaban los postreros deseos del futuro difunto, su "última voluntad".

A propósito del mobiliario, constatamos que siete de cada diez inventarios consultados hacen referencia a muebles para dormir, ya fueran camas, cujas, catres o marquesas. Proporción contundente que, estimamos, en la realidad era aún mayor. Si en algunas listas de bienes no hay referencias a muebles para dormir ¿significaba que en esa casa no había camas? Si así fuera, esperaríamos este vacío en las viviendas de las familias de los sectores subalternos y más pobres. Sin embargo, en los inventarios de prósperos comerciantes como Tiburcio Valeriano Húmeres, José

\footnotetext{
${ }^{39}$ Manuel Carreño, Manual de urbanidad... Op. Cit., p. 51

40 Michelle Perrot. "Formas de Habitación" en Georges Duby y Michelle Perrot (directores) Historia de la vida privada T. 4 De la Revolución Francesa a la Primera Guerra Mundial. Madrid, Taurus, 2005, pp. 301, 302.

${ }^{41}$ Eric Hobsbawm. La era del capital, 1848-1875. Buenos Aires, Crítica, 2010, p. 239.

42 Archivo del Arzobispado de Córdoba, Causas matrimoniales, Juicios de divorcio, Años 1811 - 1814 , tomo VIII, leg. 201, exp. 16, f. 1 vto. Citado por Mónica Ghirardi. Matrimonios y familias en Córdoba. Prácticas y representaciones. Córdoba, Universidad Nacional de Córdoba, 2004, p. 301.
} 


\section{Cecilia Moreyra}

Manrique de Lara, Simón Gutiérrez y Andrés Castellanos, ${ }^{43}$ no se menciona mobiliario para dormir. ¿Carecían de camas estas residencias o, por algún motivo, se eludió mencionarlas en el inventario? ¿se concentró el tasador en registrar los bienes económicamente más valiosos como terrenos, casas, animales o mercaderías, en detrimento de objetos menos importantes? A este respecto, el reclamo que realiza Francisca de León hecha algo de luz sobre esta ¿llamativa? inexistente mención de camas en algunos documentos. Francisca solicitó se revisaran dos errores que, a su juicio, habían cometido quienes inventariaron los bienes de su difunto marido Manuel Hurquiri. En primer lugar, valuaron muchos de los bienes en precios sumamente elevados y en segundo, cometieron el desacierto de incluir en la lista de bienes "el lecho y la humilde ropa del mismo" 44 . Tal reclamo sugiere que, en muchos casos, el lecho cotidiano no se incluía (o no debía incluirse) en el inventario, no por escasez de valor económico sino, más bien, porque los sujetos le otorgaron valoración simbólica, de allí que nos encontremos con total ausencia de referencias a mobiliario para dormir en algunos documentos, incluidos los inventarios de personas de los sectores acomodados. Por otra parte, hay registros que, sin citar cama alguna, sí mencionan colchón y ropa de cama ${ }^{45}$, por lo que es probable que algunas personas durmieran sobre un colchón o jergón colocado directamente sobre el piso o un banco de madera. ${ }^{46}$ Ello da cuenta de un lecho ciertamente móvil. Los esclavos y sirvientes, por su parte, dormían directamente sobre el suelo de la cocina, patio o azotea, según observó William McCann en su paso por la ciudad de Córdoba. En la vivienda en que se alojara el viajero inglés, eran los patios y azoteas los lugares donde dormía la servidumbre femenina. ${ }^{47}$

A la hora del reposo nocturno, de la siesta, del parto, de la sexualidad o de la enfermedad, las personas se recostaban en cujas, marquesas o catres. El vocablo "cama" aludía al conjunto de colchón o jergón, junto con sábanas, almohadas y colchas. Así se expresó en el inventario de María del Rosario Arguello, en cuyo aposento había "una cuja con su cama"48. La cama podía ir directamente sobre el piso o sobre un mueble de madera o hierro denominado cuja o marquesa. En efecto, el Diccionario de la Academia Usual en su edición de 1803, define el término "cama" de la siguiente manera: "el lecho [compuesto de] xergón, colchón, sábanas, manta y colcha; todo lo cual puede estar en el suelo y se llama cama; pero comúnmente se

\footnotetext{
43 "Inventario de Tiburcio Valeriano Húmeres", Córdoba, 1811, AHPC, Esc. 1, Leg. 440, exp.1; "Inventario de José Manrique de Lara", Córdoba, 1818, AHPC, Esc. 1, Leg. 450, Exp.5; "Inventario de Simón Gutiérrez", Córdoba, 1828, AHPC, Esc. 3, Leg. 82, Exp.3; "Inventario de Andrés Castellanos", Córdoba, 1845, AHPC, Esc. 4, Leg. 92, Exp. 11.

44 "Inventario de Manuel Hurquiri”, Córdoba, 1811, AHPC, Esc. 1, Leg, 440, Exp. 20, f. 209.

45 Tal es el caso del pardo Manuel Hurquiri, ("Inventario de Manuel Hurquiri", Córdoba, 1811, AHPC, Esc.1, Leg. 440, Exp.20, f. 197) y del pulpero Anselmo Flores ("Inventario de Anselmo Flores", Córdoba, 1848, AHPC, Esc. 1, Leg. 480, Exp.1, f. 4v).

46 Miguel Antonio Fernández, por ejemplo, contaba con "un par de bancos ordinarios que sirven para cama", "Inventario de Miguel Antonio Fernández", Córdoba, 1791, AHPC, Esc. 1, Leg. 412, Exp. 1, f. $21 \mathrm{v}$.

47 William Mac Cann. Viaje a caballo por las provincias argentinas. en Carlos Segreti. Córdoba. Ciudad y provincia... Op. Cit., p. 368.

48 “Testamentaria de María del Rosario Argüello", Córdoba, 1850, AHPC, Esc. 4, Leg. 98, Exp. 20, f. s/d.
} 


\section{La alcoba, el lecho, lo cotidiano. Cultura material de un espacio doméstico. Córdoba (Argentina), siglos XVIII y XIX}

pone sobre alguna armazón de madera o hierro, que por sí sola o junta con la ropa se llama también cama". ${ }^{49}$

Las cujas tenían armazón de madera de algarrobo, caoba, jacarandá, veta, naranjo, nogal o pino, o bien podían ser de hierro o bronce. Las columnas o pilares que tenían en sus cuatro esquinas, sostenían el cielorraso de cuero o tela y las colgaduras o cortinas que, al correrse, protegían contra el frío y los insectos y resguardaban de miradas ajenas, construyendo, como ya referimos, ciertas experiencias de intimidad y privacidad. Las cujas podían albergar a una o dos personas y eran designadas, respectivamente, "cuja de soltero" 50 y "cuja matrimonial;"51 denominación que asociaba el tamaño del mueble con el estado conyugal de las personas que en ella dormían. Así, el soltero dormiría solo y los casados, juntos. Esta distinción entre cuja de soltero y matrimonial alude a la concepción según la cual la sexualidad, que se propiciaba si dos personas dormían juntas, era considerada legítima si se daba en el marco del matrimonio. Sobra decir que no se trata más que de construcciones discursivas que no se correspondían con la realidad. Pero, aun sin ajustarse a las prácticas concretas, estos términos reproducían las representaciones sociales en torno a la sexualidad legítima.

En cuanto a las denominadas marquesas, éstas eran armazones o "marcos" sobre los que se colocaba el lecho; se distinguían de las cujas por la ausencia de pilares, cielorraso y cortinas. Sus dimensiones $-1,80$ metros de largo por 1 metro de ancho- sugieren que eran usadas por una sola persona, algo similar a la "cuja de soltero".

Más que cujas y marquesas, el mueble habitual para recostarse era el catre, una "cama ligera", cuyas piezas se doblaban "para poderse llevar y usar cómodamente", 52 sus patas tenían forma de tijera, lo que facilitaba su pliegue para el trasladado. Este tipo de cama era el que se usaba en las campañas militares que exigían desplazamiento constante: "un catre de campaña de tres piezas" llevaba consigo el Teniente Coronel Gil Domínguez. ${ }^{33}$ Dentro del catre, el espacio destinado a recostar el cuerpo, ya fuera con o sin colchón, era de cuero, lona o tabla. Cujas y catres producían experiencias corporales diferentes, recostarse en uno u otro mueble podía ser más o menos confortable; la superioridad numérica de los catres, por sobre las cujas y marquesas, evidencia el restringido acceso al reposo más confortable. Si ensayamos una suerte de jerarquía de muebles para dormir, tenemos a los catres como el mueble más básico, en que dormía el personal de servicio; esto, claro, cuando no se acostaban directamente sobre el suelo. Joaquín de Oliveira, por ejemplo, contaba con "dos catres de madera para cama de criados." ${ }^{4}$ El catre

\footnotetext{
${ }^{49}$ Diccionario de la Academia Usual. Madrid, Real Academia Española, 1803, p. 160.

50 "Sucesorio de Xaviera Torres", Córdoba, 1836, AHPC, Esc.2, Leg. 126, Exp.30, f. 2.

51 "Inventario, tasación y partición de Juan Borton", Córdoba, 1820, AHPC, Esc. 1, Leg, 453, Exp. 13, f.

6; "Inventario de Pablo Cires", Córdoba, 1822, AHPC, Esc. 2, Leg. 116, Exp.12, f. 5.

52 Diccionario de la Academia Usual, (Madrid: Real Academia Española, 1822), 173.

53 "Inventario de Gil Domínguez", Córdoba, 1829, AHPC, Esc. 3, Leg. 84, Exp.5, f. s/d.

54 "Inventario, tasación y partición de Joaquín Xavier de Oliveira", Córdoba, 1811, AHPC, Esc. 1, Leg. 450, Exp.9, f. 4v.
} 


\section{Cecilia Moreyra}

representa, a su vez, el original carácter mueble (móvil) del mobiliario, es decir, artefactos que se llevaban de un lugar a otro, de una habitación a otra, lo que demuestra la esencial provisionalidad de muchos lechos cotidianos.

A pesar de las diferencias entre cujas y catres en términos de comodidad, siempre será más confortable recostarse sobre alguno de estos muebles antes que en el suelo, sin mediar más que un jergón o un trozo de cuero. Esta última era la situación corriente de los esclavos y las personas más pobres que, según el relato de Thomas Hutchinson, "no tienen más cama sobre que dormir que los montones de vainas de algarroba, guardada para provisiones del invierno". Al pasar por el "puesto de Castro", en el departamento de Rio Seco, este viajero describió la manera en que vivía una familia pobre del lugar. Aunque el relato se emplaza en el espacio rural, bien vale de ejemplo para formarnos una imagen de cómo dormían las personas en situación de pobreza. Hutchinson declara que "no hay adentro nada en forma de cama a no ser un cuero de vaca, en el que duermen el padre, la madre, siete hijos y un muchacho huérfano [...] una familia compuesta por diez personas, todas juntas en montón". ${ }^{55}$ Esta disposición corporal y espacial, a la hora de dormir, era la mejor manera de defenderse del frío y de eventuales ataques de animales. Por otra parte, esta situación de contacto también engendraba un contexto doméstico que favorecía el desarrollo y contagio de múltiples enfermedades y conformaba, a su vez, un espacio de promiscuidad. El resultado era una débil separación entre adultos y niños, hombres y mujeres, enfermos y sanos, vivos y muertos. El carácter multiactoral del lecho encuentra aquí, su punto máximo de expresión.

Cujas, marquesas y catres se elevaban algún trecho por encima del nivel general del suelo, dando lugar a un espacio debajo donde tenían lugar objetos vinculados a prácticas vitales: las bacinicas, artefactos de forma redonda más o menos profunda con un asa a un lado que permitía su manipulación. En su cavidad se contenían las evacuaciones diarias de los usuarios de la habitación, desechos que eran luego arrojados en los lugares comunes, ese pequeño cuarto emplazado entre los espacios de servicio. De este modo, a la diversidad de experiencias cotidianas que se vivían en las alcobas, se anexa la satisfacción de las necesidades fisiológicas, confirmando, pues, la versatilidad de los espacios domésticos y las dispares fronteras de sensibilidad.

Finalmente, aunque aparecen en exiguas cantidades, resulta relevante las referencias a cunas o camas para niños en los inventarios. Entre los documentos elaborados durante la década de 1810, contabilizamos solo un ejemplar de este tipo de objetos. En cambio, avanzado el siglo XIX, hacia 1850, identificamos siete cunas o camas para niños. Estos cortos números pueden constituir indicios de incipientes cambios en la organización doméstica a la hora de dormir que expresaba la tendencia hacia la individualización de los bebés y niños de la casa. La construcción, comercialización y uso de muebles específicamente pensados para los menores de

55 Thomas J. Hutchinson. Buenos Aires y otras provincias argentinas. Buenos Aires, 1945, en Carlos Segreti Córdoba. Ciudad y provincia... Op. Cit., pp. 425, 430, 431. 


\section{La alcoba, el lecho, lo cotidiano. Cultura material de un espacio doméstico. Córdoba (Argentina), siglos XVIII y XIX}

la familia, indicarían una creciente atención otorgada a los niños. En la casa de Rafael Barboza, por ejemplo, no solo había camas, sino también asientos destinados exclusivamente a sus hijas menores: "el sillón del uso de Lucía” y "el sillón de uso de Margarita."56 La modesta presencia de cunas o camas destinadas para los niños en la Córdoba del siglo XIX, puede señalar una incipiente individualización y autonomización del infante dentro de la familia, tendencia que se insertó en un marco más amplio que se extiende desde fines del siglo XVIII y el siglo XIX e involucra Europa, Latinoamérica y, también, la región rioplatense. Para el espacio europeo, Philippe Ariés reconoce que recién avanzada la edad moderna el niño comienza a ser objeto de una consideración especial. Hasta ese momento, la infancia no era interpretada como una edad diferenciada de la vida adulta ${ }^{57}$. Lawrence Stone sitúa ese cambio de actitud hacia los niños en la Inglaterra del siglo XVIII. Ello implicó nuevas prácticas en cuanto a la crianza y educación de los niños y las nuevas relaciones entre hijos y padres se expresaron en objetos materiales como la ropa, libros para niños y juegos didácticos. ${ }^{58}$ En Argentina, Ricardo Cicierchia identifica un creciente interés por parte de la medicina y de la justica del siglo XIX, hacia la minoridad. Desde estos espacios se intentó atender a la crianza y la educación de los niños y también a cuestiones aún más complejas como el abandono de menores, el infanticidio y la entrega de niños. ${ }^{59}$

\section{Los textiles}

Sobre cujas y catres iba el lecho compuesto por colchón, sábanas, almohadas, fundas, colchas y sobrecamas. El colchón permitía, como expresaba el Diccionario de las Autoridades de 1729, "acostarse blandamente" 60 , produciendo mayor confort que al dormir sobre una tabla rígida o directamente sobre el piso. Los colchones estaban rellenos con paja o lana y forrados con lienzo, cotín, algodón o brin. Los de lana perjudicaban la salud al "conservar las exhalaciones y vapores malignos o abrasar de calor a los enfermos". Se pensaba que otros elementos, como la cerda, algodón, estopa, pluma, hojas de maíz o paja seca eran mejores, más frescos y no tan peligrosos como la lana. ${ }^{61}$

Las sábanas que envolvían el colchón entraban en contacto directo con el cuerpo, ya sea que este durmiera con prendas especiales para dormir o, simplemente, desnudo. Las sábanas se confeccionaban, en su mayoría, con géneros delgados hechos de lino

\footnotetext{
56 “Juicio Testamentario de Rafael Barboza, Córdoba, 1851, AHPC, Esc. 1, Leg. 486, Exp. 5, f. 8.

57 Philippe Ariès. El niño y la vida familiar en el Antiguo Régimen. Madrid, Taurus, 1987.

58 Lawrence Stone. Familia, Sexo y matrimonio en Inglaterra 1500-1800. México, Fondo de Cultura Económica, 1990. Cfr. Sharon Brookshaw. "The Material Culture of Children and Childhood: Understanding Childhood Objects in the Museum Context". Journal of Material Culture Vol. 14, $\mathrm{N}^{\circ} 3$, 2009, pp. 365-383.

${ }^{59}$ Ricardo Cicerchia. Historia de la vida privada en Argentina. Buenos Aires, Troquel, 1998, pp. 75-86.

60 Diccionario de las Autoridades. Madrid, Real Academia española, 1729, p. 407.

61 Semanario de Agricultura, Industria y comercio, enero de 1807, f. 123, 124 y 134-136. Citado por Nelly Porro, Juana Astiz y María Róspide. Aspectos de la vida cotidiana en el Buenos Aires Virreinal. Buenos Aires, Universidad de Buenos Aires, 1982, p. 61.
} 


\section{Cecilia Moreyra}

o cáñamo, tales como bramante, cambray, hilo, lienzo y estopilla. Telas asimismo utilizadas en la confección de camisas, enaguas y calzoncillos. Al igual que éstas, las piezas textiles que conformaban el lecho se asentaban bajo el rubro "ropa blanca". La mayoría de las sábanas registradas eran de este tipo de géneros, algunas pocas, de algodón y solo ciertas piezas, especialmente en las primeras décadas del siglo XIX, contaban con algún tipo de guarnición o adorno en forma de volados o encajes de seda. Entre las almohadas y fundas, también predominaron los géneros livianos hechos de lino o cáñamo y, aunque en menor medida, también se presentan telas de algodón. En estas piezas se observan, con mayor frecuencia que en las sábanas, algunos volados, encajes y bordados que otorgaban cierta distinción al lecho en su conjunto. Las colchas o sobrecamas iban encima de sábanas y frazadas cumpliendo una función de abrigo y, gracias a los flecos, bordados y vuelos, también adornaban la cama. Eran, en su mayoría, de telas de algodón, principalmente zaraza, y las había, también, de seda o lana.

En las cartas de dote encontramos a las mujeres ingresando al matrimonio colchón, sábanas, almohadas y fundas. Conseguir un conveniente ajuar de cama era el ideal de muchas novias, en especial, aquellas con altas posibilidades económicas. Josefa Gutiérrez, por ejemplo, llevó como dote al casarse con el coronel de los Reales Ejércitos Francisco Xavier Tirry, la notable suma de 29.436 pesos. Entre los numerosos bienes que componían la dote, se destacan los objetos para preparar un confortable, y hasta ostentoso, lecho matrimonial: una colgadura de cama con rodapié y colcha forrados y flecadura de seda carmesí, todo de damasco nuevo, tasado en 292 pesos; cuatro fundas de almohada de tafetán nácar en siete pesos; tres piezas de hilera para presilla de dicha colgadura; fundas de almohada de coleta aplomadas en seis pesos; un colchón nuevo de buenos colores en doce pesos. ${ }^{62}$ Avanzado el siglo XIX, continuamos observando esta particularidad en cuanto a los bienes que ingresaban las mujeres al matrimonio. Manuela Irazoque entregó como dote a su hija un colchón de cotín; tres sábanas de bramante; dos almohadas; dos fundas de hilo bordadas de encajes; dos fundas de algodón; una frezada; una colcha de zaraza y otra de algodón. ${ }^{63}$ La novia llevando colchón y sábanas a la morada conyugal era una práctica que ya se observa en la Europa moderna64. Como escenario de la sexualidad legítima, el lecho era el símbolo del matrimonio y la vida en pareja. Así, la cópula conyugal llevada a cabo en la cama y alcoba, era el inicio simbólico del matrimonio y la familia. En este marco, la impotencia sexual que significaba la imposibilidad de procrear, objetivo sustancial de la sociedad conyugal, fue considerada una justificada causa de nulidad matrimonial ${ }^{65}$.

Resulta significativo que a medida que avanzaba el siglo XIX, la ropa de cama dejaba de incluirse dentro del inventario de bienes. Así, siete de cada diez documentos llevados a cabo durante la década de 1810 registran colchones, sábanas, almohadas,

\footnotetext{
62 “Dote de Josefa Gutiérrez”, Córdoba, 1785, AHPC, Registro 1, Inv. 168, f. 114v

63 "Inventario de Manuela Irazoque”, Córdoba, 1865, AHPC, Esc. 2, Leg. 164, Exp.16, f. 12v.

${ }^{64}$ Rafaella Sarti. Vida en familia... Op. Cit., pp. 66, 67.

65 Mónica Ghirardi. Matrimonios y familias...Op. Cit., p. 239.
} 


\section{La alcoba, el lecho, lo cotidiano. Cultura material de un espacio doméstico. Córdoba (Argentina), siglos XVIII y XIX}

fundas, etc.; relación que va decreciendo, hasta encontrarnos, en la década de 1860, con cuatro inventarios de cada diez que registran algún tipo de ropa de cama. Concluimos, pues, que decreció la importancia otorgada a este rubro en las tasaciones de bienes de difuntos. Cuando el proceso de industrialización europeo, y el consecuente crecimiento del comercio, provocaron un aumento de los bienes disponibles para el consumo, se acortó la vida útil de muchos objetos, los que paulatinamente dejaron de circular de generación en generación y de ser continuamente reciclados. Esto impactó en la inclusión o exclusión de determinados bienes al hacer las correspondientes tasaciones y particiones. En tanto las herencias giraban en torno a los bienes inmuebles y semovientes, otros objetos, como la ropa de casa o prendas de vestir, antes presentes en cada testamento o inventario, se convirtieron en rubros secundarios al interior de las tasaciones y particiones de bienes. Lo anterior también se evidencia al considerar el estado de la ropa de cama. Mientras que en la década de 1810 la mayoría de los objetos que conformaban el lecho se registraron como "usados", avanzado el siglo XIX, pocas piezas de la ropa de cama fueron consignadas como usadas o gastadas. Sin duda, a medida que se extendió el acceso a colchones, sábanas, almohadas y colchas nuevas, compradas en tiendas de la ciudad o confeccionadas con telas adquiridas en tales establecimientos, estas piezas dejaron de circular entre generaciones mediante herencias y dotes.

\section{Conclusiones}

A lo largo del trabajo observamos una habitación de las viviendas de la ciudad de Córdoba. Acotar la mirada a un fragmento del espacio doméstico resultó una singular vía para pensar las formas de organización de los espacios interiores y sus prácticas cotidianas. Hicimos foco en las materialidades de esos cuartos, es decir, sus características físicas y los muebles y objetos que los habitaban. Es en las interacciones entre estas materialidades y los sujetos, que se producen múltiples formas y sentidos de lo cotidiano. Ello implica reconocer las agencias de diferentes actores: personas, pero también, cosas. Un particular tipo documental resultó esencial para aproximarnos al espacio doméstico: los inventarios postmortem. Sus extensas y exhaustivas nóminas de los objetos que poblaban las viviendas, así como las especificaciones respecto a las características edilicias de esas residencias, coadyuvaron en la construcción de un panorama general de algunas alcobas y lechos. Cuantificar algunos objetos propios de estos cuartos y reconocer su frecuencia a lo largo del tiempo, posibilitó la identificación de algunos cambios, así como permanencias, en las formas de organizar los interiores domésticos. Las fuentes permitieron, también, la producción de acabadas descripciones de estas habitaciones. Operación básica para trascender el pensamiento convencional, habitual a la hora de investigar el universo de lo cotidiano.

El período observado es amplio, con más o menos acelerados cambios en el orden de lo político y económico, ya para el espacio rioplatense, ya para la ciudad de 


\section{Cecilia Moreyra}

Córdoba. Frente a tales procesos dinámicos, el universo de lo cotidiano parece sumido en la inmutabilidad. Pero, aun siendo lentos y a veces imperceptibles, los cambios en el espacio doméstico son un hecho. Así, durante este período reconocimos indicios de especialización de las estancias de la casa, más visibles, por supuesto, en las viviendas de mayores dimensiones, es decir, las casas organizadas en torno a dos o tres patios centrales. Sin embargo, aun dentro de una tendencia hacia la especialización e individualización del espacio doméstico, en las alcobas convivían diversidad de objetos que encarnaban múltiples actividades que no se reducían al reposo nocturno. Era, pues, la alcoba escenario de la sexualidad, de la enfermedad, de la muerte, de violencia doméstica, de trabajo y de la satisfacción de necesidades fisiológicas.

De la diversidad de prácticas e interacciones que se desarrollaban en las alcobas emergen ciertos sentidos y significados que encarnaba este espacio. Así, la "cama matrimonial" se instituyó como el espacio símbolo de la vida conyugal y su ejercicio legítimo de la sexualidad, cuya finalidad era la reproducción. Eran, pues, la alcoba y la cama símbolos de la vida en pareja y, con ello, de la familia. Estaba claro que cuando se producía "separación de cuerpos", es decir, la disolución de lazo matrimonial, se entendía que ello implicaba, antes que nada, "separación de lecho"66. Signos de incipiente individualización en la organización hogareña de los cuerpos, fueron los pocos, pero significativos ejemplares de cunas o camas para niños registradas en los inventarios. Ello evidencia una progresiva atención otorgada a la infancia, al bebe o niño, como sujeto que ocuparía espacios y objetos específicos, en este caso, un lugar para dormir. Lo anterior forma parte de un proceso más amplio de individualización y especialización, expresado en la producción y uso de objetos cada vez más específicos, destinados a funciones diferenciadas unas de otras. Esto mismo ocurría, por ejemplo, con el mobiliario de guardado: a los multifuncionales y versátiles baúles y cajas se sumaron, a lo largo del siglo XIX, muebles pensados, fabricados y usados para guardar cosas específicas, tal es el caso de las cómodas y roperos para guardar ropa o los armarios para guardar loza ${ }^{67}$.

En una sociedad donde primaban las diferencias socio-étnico-económicas, los entornos materiales cotidianos no eran en absoluto uniformes. Así, vimos familias compartiendo un trozo de cuero en el suelo de la casa, juntos, amontonados, protegiéndose del frío, de los ataques, rozando sus cuerpos, compartiendo enfermedades así como acercamientos sexuales. Observamos esclavos durmiendo en el suelo de la cocina, en el patio y, en el mejor de los casos, en un "cuarto para criados" estratégicamente ubicado en los espacios de servicio. Esa tendencia hacia la especialización de los espacios y los objetos no se visualiza entre los grupos subalternos, aquellas casas compuestas de un solo cuarto implicaban que un mismo lugar hacía las veces de dormitorio, cocina, comedor y lugar de trabajo. Por otra parte, en las casas a patios, las alcobas formaban parte de los espacios principales

${ }^{66}$ Mónica Ghirardi, Matrimonios y familias...Op. Cit., p. 296.

${ }^{67}$ Cecilia Moreyra. "En busca del confort cotidiano. El mobiliario doméstico en Córdoba (Argentina), siglo XIX". Anuario de Historia Regional y de las Fronteras, Vol. 23 No. 1, pp. 73-91. 


\section{La alcoba, el lecho, lo cotidiano. Cultura material de un espacio doméstico. Córdoba (Argentina), siglos XVIII y XIX}

colindando, muchas veces, con la sala principal y otros cuartos con los que estaban comunicados a través de puertas en común. En esta disposición espacial no se vislumbran fronteras estrictas que diferencien espacios privados e íntimos de los que no los son; para esta época el proceso de privatización de los ambientes de la casa es una tendencia aún incipiente.

\section{Bibliografía}

Appadurai, Arjun (Editor). La vida social de las cosas. Perspectiva cultural de las mercancías. México, Grijalbo, 1991.

Ariès, Philippe y Duby, Georges (Directores). Historia de la vida privada, 5 tomos. Barcelona, Taurus Minor, 2001.

Ariès, Philippe. El niño y la vida familiar en el Antiguo Régimen. Madrid, Taurus, 1987.

Ayala Alonso, Enrique. "Habitar la casa barroca. Una experiencia en la ciudad de México" Actas del III Congreso Internacional del Barroco, 2001.

Bauer, Arnold. Goods, Power, History. Latinamerica's material culture. Nueva York, Cambridge University Press, 2001.

Becker, Howard. Trucos del oficio. Cómo conducir su investigación en ciencias sociales. Buenos Aires, Siglo XXI Editores, 2009.

Braudel, Fernand. Civilización material, economía y capitalismo, siglos XV-XVIII. 1. Las estructuras de lo cotidiano. Madrid, Alianza, 1984.

Brookshaw, Sharon. "The Material Culture of Children and Childhood: Understanding Childhood Objects in the Museum Context". Journal of Material Culture Vol. 14, N³, 2009, pp. 365-383.

Burmeister, Hermann. Viaje por los Estados del Plata. Buenos Aires, 1944. En Córdoba. Ciudad y provincia siglo XVI-XX. Según relatos de viajeros y otros testimonios, compilación de Carlos Segreti. Córdoba, Junta Provincial de Historia de Córdoba, 1973, pp. 402-419.

Carreño, Manuel. Manual de urbanidad y buenas maneras, para uso de la juventud de ambos sexos, en el cual se encuentran las principales reglas de civilidad y etiqueta que deben observarse en las diversas situaciones sociales, precedido de un breve tratado sobre los deberes morales del hombre. Córdoba, Buena Vista, 2010. 


\section{Cecilia Moreyra}

Cicerchia, Ricardo. Historia de la vida privada en Argentina. Buenos Aires, Troquel, 1998.

Corbin, Alain. El perfume o el miasma. El olfato y lo imaginario social. Siglos XVIII y XIX. México, Fondo de Cultura Económica, 2002.

Dejean, Joan. The Age of comfort. When Paris Discovered Casual and the Modern Home Began. Nueva York, Bloomsbury USA, 2010.

Dibie, Pascal. Etnología de la Alcoba. El dormitorio y la gran aventura del reposo de los hombres. Barcelona, Gedisa, 1989.

Diccionario de la Academia Usual. Madrid, Real Academia Española, 1803.

Diccionario de la Academia Usual. Madrid, Real Academia Española, 1822.

Diccionario de las Autoridades. Madrid, Real Academia española, 1729.

Eizaguirre, José Manuel. Córdoba. Primera serie de cartas sobre la vida y costumbres en el interior. Córdoba: R. Bruno y Ca. Editores, 1898.

Elias, Norbert. El proceso de la civilización. Investigaciones sociogenéticas y psicogenéticas. México, Fondo de Cultura Económica, 2009.

Elias, Norbert. La sociedad cortesana. México, Fondo de Cultura Económica, 2012.

Franco Rubio, Gloria. "La vivienda en el Antiguo Régimen. De espacio habitable a espacio social”. Chronica Nova N³5, Granada, 2009, pp. 63-103.

García González, Francisco. "Vida cotidiana y cultura material en el Zacatecas colonial" en Gonzalobo Aizpuru, Pilar (dir): Historia de la vida cotidiana en México vol III el siglo XVIII: entre tradición y cambio, FCE, México, 2005, pp.45-71.

García Montes, Luis. "Medidas antiguas: la vara." Toletum: boletín de la Real Academia de Bellas Artes y Ciencias Históricas de Toledo N27, Toledo, 1991, pp. 153160.

Ghirardi, Mónica. Matrimonios y familias en Córdoba. Prácticas y representaciones. Córdoba, Centro de Estudios Avanzados, 2004.

Glassie, Henrry. Material culture. Bloomington/Indianapolis, Indiana University press, 1999.

Hobsbawm, Eric. La era del capital, 1848-1875. Buenos Aires, Crítica, 2010.

Hutchinson, Thomas J. Buenos Aires y otras provincias argentinas. Buenos Aires, 1945. En Córdoba. Ciudad y provincia siglo XVI-XX. Según relatos de viajeros y otros 
La alcoba, el lecho, lo cotidiano. Cultura material de un espacio doméstico. Córdoba (Argentina), siglos XVIII y XIX

testimonios, compilación de Carlos Segreti. Córdoba, Junta Provincial de Historia de Córdoba, 1973, pp. 420-447.

Lins Ribeiro, Gustavo. "Descotidianizar. Extrañamiento y conciencia práctica, un ensayo sobre la perspectiva antropológica". En Mauricio Boivin, Ana Rosato y Victoria Arribas (editores) Constructores de otredad. Una introducción a la antropología social y cultural. Buenos Aires, Eudeba, 1998, pp. 194-198.

Mac Cann, William. Viaje a caballo por las provincias argentinas. En Córdoba. Ciudad y provincia siglo XVI-XX. Según relatos de viajeros y otros testimonios, compilación de Carlos Segreti. Córdoba, Junta Provincial de Historia de Córdoba, 1973, pp. 360-368.

Mintz, Sindey. Dulzura y poder. El lugar del azúcar en la historia moderna. México, Siglo XXI editores, 1996.

Morales Tejeda, Aida. El signo francés en Santiago de Cuba. Santiago de Cuba, Editorial Oriente, 2015.

Moreyra, Cecilia. "Cultura material e higiene cotidiana en la Córdoba del Ochocientos". Anuario de Estudios Americanos, Vol. 74, № 1, Sevilla, 2017, pp. 211234.

Moreyra, Cecilia. "En busca del confort cotidiano. El mobiliario doméstico en Córdoba (Argentina), siglo XIX". Anuario de Historia Regional y de las Fronteras, Vol. 23 No. 1, pp. 73-91.

Moya Gutiérrez, Arnaldo. “Cultura material y vida cotidiana: el entorno doméstico de los vecinos principales de Cartago (1750-1820)" en Molina Jiménez, Iván y Palmer, Steven (Ed) Héroes al gusto y libros de moda. Sociedad y cambio cultural en Costa Rica (1750/1900), San Jose, Costa Rica, Ed. Porvenir, Plumsock Mesoamerican Studies, 1992.

Otero, Osvlado. La vivienda porteña en tiempos virreinales, La vivienda porteña en tiempos virreinales. Materiales, uso, función y valor simbólico. Tesis de doctorado en Historia. La Plata, Universidad Nacional de La Plata, 2004.

Pérez Salas, María Esther. "El trajín de una casa” en Gonzalbo Aizpuru Pilar (Dir): Historia de la vida cotidiana en México Vol. IV: Bienes y vivencias. El siglo XIX, El Colegio de México y FCCE, México DF, 2005. pp. 179-212.

Perrot, Michelle. "Formas de Habitación". En Georges Duby y Michelle Perrot (directores) Historia de la vida privada T. 4 De la Revolución Francesa a la Primera Guerra Mundial. Madrid, Taurus, 2005, pp. 301-316.

Perrot, Michelle. Historia de las alcobas. México, Fondo de Cultura Económica, 2011. 


\section{Cecilia Moreyra}

Porro, Nelly, Astiz, Juana y Róspide, María. Aspectos de la vida cotidiana en el Buenos Aires Virreinal. Buenos Aires, Universidad de Buenos Aires, 1982.

Roche, Daniel. La Culture des apparences: essai sur l'histoire du vêtement aux ss. XVII y XVIII. París, Fayard, 1989.

Sarti, Rafaella. Vida en familia. Casa, comida y vestido en la Europa moderna. Barcelona, Crítica, 2003.

Stone, Lawrence. Familia, Sexo y matrimonio en Inglaterra 1500-1800. México, FCE, 1990

Woodward, Ian. Understanding material culture. Londres, SAGE Publications, 2007.

\section{Fuentes Primarias}

Archivo Histórico de la Provincia de Córdoba (AHPC), Córdoba, Argentina, Escribanías $n^{\circ} 1,2,3$ y 4.

Archivo Histórico de la Provincia de Córdoba (AHPC), Córdoba, Argentina, Protocolos Notariales, Registro 1.

Recibido: 20/09/2018

Evaluado: $18 / 10 / 2018$

Versión Final: 26/11/2018 